\title{
Association of NAFLD Fibrosis Score with \\ Subclinical Myocardial Remodeling in Patients with Type 2 Diabetes: A Cross-Sectional Study in China
}

Nengguang Fan

Shanghai Jiao Tong University

Xiaoying Ding

Shanghai Jiao Tong University School of Medicine

$\mathrm{Na} \mathrm{Li}$

Shanghai Jiao Tong University

Qin Zhen

Shanghai Jiao Tong University School of Medicine

\section{Liping Gu}

Shanghai Jiao Tong University School of Medicine

\section{Fang Fang}

Shanghai Jiao Tong University

\section{Shuai Yan}

Shanghai Jiao Tong University

Lin Pan

Shanghai Jiao Tong University

\section{Aifang Zhang}

Shanghai Jiao Tong University School of Medicine

Tingting Shen

Shanghai Jiao Tong University School of Medicine

\section{Yufan Wang}

Shanghai Jiao Tong University

Yongde Peng ( $\nabla$ yongdepeng0908@126.com )

\section{Original investigation}

Keywords: Nonalcoholic fatty liver disease fibrosis score, Type 2 diabetes mellitus, Subclinical myocardial remodeling

Posted Date: June 9th, 2020

DOI: https://doi.org/10.21203/rs.3.rs-33373/v1 
License: (c) (i) This work is licensed under a Creative Commons Attribution 4.0 International License. Read Full License 


\section{Abstract}

Background: Nonalcoholic fatty liver disease (NAFLD), especially with liver fibrosis, is associated with cardiovascular diseases (CVD). NAFLD fibrosis score (NFS), a noninvasive marker of advanced fibrosis first developed in patients with NAFLD, has found to be associated with CVD in different populations including with diabetes, regardless of the presence of NAFLD. The aim of the present study was to determine whether NFS is associated with subclinical myocardial remodeling in type 2 diabetic patients.

Methods: A cross-sectional study was performed in type 2 diabetic patients. NFS derived from available parameters was calculated and the subjects were divided into four groups according to the quartiles of NFS. Subclinical myocardial remodeling was examined by echocardiography and its association with NFS was analyzed.

Results: A total of 1878 type 2 diabetic patients were enrolled in the present study. Subjects with higher NFS were older, had a longer duration of diabetes and higher levels of body mass index (BMI), systolic blood pressure (SBP) and serum creatinine (Scr) than those with lower NFS $(P<0.01)$. Multiple linear regression analysis revealed that sex, age, BMI, LDL-C and HbA1c were independent determiners of NFS in type 2 diabetic patients. Parameters of subclinical myocardial remodeling including left atrial dimension (LAD), interventricular septum thickness (IST), left ventricular end-diastolic diameter (LVEDD), left ventricular end-systolic diameter (LVESD) and left ventricular posterior wall thickness (LVPWT) were all gradually increased with the increment of NFS. Linear regression analysis further revealed that NFS was positively associated with LAD, IST, LVEDD, LVESD and LVPWT after adjustment for the confounding factors.

Conclusions: NFS is independently associated with subclinical myocardial remodeling in type 2 diabetic subjects.

\section{Background}

Nonalcoholic fatty liver disease (NAFLD), defined as excessive accumulation of triglycerides in hepatocytes with no evidence of alcohol abuse or other secondary causes, has become the most prevalent liver diseases worldwide[1]. It represents a spectrum of conditions from simple steatosis to nonalcoholic steatohepatitis (NASH), liver fibrosis and cirrhosis, and has been one of the top leading causes for end-stage liver disease and liver transplantation[2].

In addition to liver-related complication, a growing body of evidence has indicated a close relationship between NAFLD and cardiovascular disease (CVD)[3-5]. Patients with NAFLD have a higher prevalence of CVD and are more likely to die from CVD than from liver-related death[4-6]. Among the categories of NAFLD, liver fibrosis is considered as the main indicator of prognosis in NAFLD and was found to be independently associated with CVD and motality in different populations[6-8]. However, the relationship between liver fibrosis and CVD in type 2 diabetic patients, who have a high prevalence of NAFLD, is limitedly investigated[9, 10]. 
The NAFLD Fibrosis Score (NFS) is a noninvasive marker widely used to predict advanced fibrosis in patients with NAFLD[11]. Evidence has indicated that NFS is associated with coronary atherosclerosis and predicts cardiovascular events and mortality in subjects with NAFLD[12-14]. Further study revealed that NFS was also associated with cardiovascular mortality in different populations, including patients with heart failure or coronary artery disease and even in the general population, regardless of the presence of NAFLD $[8,13-16]$. However, the relationship between NFS and CVD in type 2 diabetic subjects was rarely studied[10, 17].

In the present study, we performed a cross-sectional investigation to determine whether NFS is associated with subclinical myocardial remodeling determined by echocardiography in type 2 diabetic subjects.

\section{Methods}

\subsection{Subjects}

All subjects were recruited from the department of Endocrinology and Metabolism in Shanghai General Hospital between May 2017 and June 2019. The diagnosis of type 2 diabetes was defined according to the 1999 World Health Organization criteria. All participants were requested to complete a standardized questionnaire that included questions on the history of present and past illnesses and medical therapies. Subjects with an alcohol intake > $140 \mathrm{~g} /$ week for men and $70 \mathrm{~g} /$ week for women, a history of viral hepatitis, auto-immune hepatitis or other forms of chronic liver disease, a history of heart failure or renal diseases were excluded from the study. Finally, a total of 1878 type 2 diabetic patients were included in final analysis. This study was approved by the Institutional Review Board of Shanghai General Hospital affiliated to Shanghai Jiao Tong University School of Medicine, and performed in accordance with the principle of the Helsinki Declaration II. Written informed consent was obtained from all subjects.

\subsection{Anthropometric and biochemical measurements}

All subjects were assessed after overnight fasting for at least $8 \mathrm{~h}$. Body weight, height, systolic and diastolic blood pressure (SBP, DBP) were measured by an experienced physician. BMI was calculated as body weight in kilograms divided by body height squared in meters.

Blood samples were collected by one experienced nurse. Fasting serum triglycerides (TG), total cholesterol (TC), low-density lipoprotein cholesterol (LDL-C), high-density lipoprotein cholesterol (HDL-C), alanine aminotransferase (ALT), aspartate aminotransferase (AST) and serum creatinine (Scr) were measured using an autoanalyzer (Beckman, Palo Alto, CA). Blood glucose were measured with glucose oxidase method. HbA1c was determined by high-performance liquid chromatography. The NAFLD fibrosis score was calculated: $-1.675+0.037 \times$ age $+0.094 \times \mathrm{BMI}+1.13+0.99 \times$ AST $/ \mathrm{ALT}-0.013 \times$ Platelet $(\times$ $\left.10^{9} / \mathrm{L}\right)-0.66 \times$ albumin $(\mathrm{g} / \mathrm{dL})$.

\subsection{Echocardiography}


Comprehensive echocardiography, including Doppler and tissue Doppler imaging, was performed using an Artida cardiac ultrasound scanner (Toshiba Medical Systems, Otawara, Japan) by trained sonographers using a standardized protocol across all field centers. Experienced sonographers made measurements from digitized images using a standard software offline image analysis system (Digisonics, TX, USA).

\subsection{Statistical analysis}

All statistical analyses were performed using SPSS 13.0 (Chicago, IL). Continuous variables were presented as means $\pm S D$ or median (interquartile range). Differences among groups were tested by oneway ANOVA for continuous variables and $x^{2}$ test for categorical variables. Pearson's correlations were performed to evaluate the associations between NFS and other metabolic risk factors, and multivariate stepwise linear regression model to identify the independent factors related to NFS. Multivariate linear regression model was also used to evaluate the independent association of NFS with parameters of subclinical myocardial remodeling. $P<0.05$ was considered statistically significant.

\section{Result}

\subsection{Clinical characteristics of the study population}

Among the 1878 type 2 diabetic patients, 623 were women and 1255 were men. Clinical and biochemical characteristics of the study population according to the quartiles of NFS were summarized in Table 1. Subjects with higher NFS were older and had a longer duration of diabetes than those with lower NFS ( $P$ values $<0.01$ ). Moreover, BMI, SBP and Scr was significantly increased while DBP, TC, LDL-C and ALT was decreased in parallel with the increment of NFS (all $P$ for trend values $<0.01$ ). No significant difference was found in gender, FPG, HbA1C, TG, HDL-C and SUA among the four groups. 
Table 1

Clinical and biochemical characteristics of the study population

\begin{tabular}{|c|c|c|c|c|c|}
\hline Variables & Q1 & Q2 & Q3 & Q4 & $P$ Value \\
\hline $\mathrm{n}$ & 469 & 470 & 470 & 469 & \\
\hline Male/female & $315 / 154$ & $330 / 140$ & 299/171 & $311 / 158$ & 0.196 \\
\hline Age (years) & $40.9 \pm 10.1$ & $47.5 \pm 10.3$ & $52.7 \pm 9.3$ & $59.8 \pm 8.3$ & $<0.001$ \\
\hline $\begin{array}{l}\text { Duration } \\
\text { (months) }\end{array}$ & $25(5-72)$ & $37(3-99)$ & $59(13-120)$ & $72(15-132)$ & $<0.001$ \\
\hline $\mathrm{BMI}\left(\mathrm{kg} / \mathrm{m}^{2}\right)$ & $25.5 \pm 3.5$ & $25.8 \pm 3.6$ & $25.6 \pm 3.5$ & $26.6 \pm 4.0$ & $<0.001$ \\
\hline $\mathrm{SBP}(\mathrm{mmHg})$ & $127.3 \pm 16.0$ & $128.5 \pm 16.5$ & $129.5 \pm 17.9$ & $132.6 \pm 17.3$ & $<0.001$ \\
\hline $\mathrm{DBP}(\mathrm{mmHg})$ & $78.9 \pm 10.0$ & $78.4 \pm 10.3$ & $77.7 \pm 10.8$ & $76.2 \pm 10.2$ & $<0.001$ \\
\hline FPG (mM) & $8.7 \pm 6.8$ & $8.4 \pm 2.9$ & $8.2 \pm 2.9$ & $8.0 \pm 2.9$ & 0.102 \\
\hline HbA1C (\%) & $8.8 \pm 2.2$ & $8.9 \pm 2.2$ & $8.7 \pm 2.1$ & $8.7 \pm 2.3$ & 0.266 \\
\hline $\mathrm{TG}(\mathrm{mM})$ & $1.9(1.4-2.6)$ & $1.6(1.1-2.5)$ & $1.5(1.0-2.1)$ & $1.5(1.1-2.2)$ & 0.082 \\
\hline $\mathrm{TC}(\mathrm{mM})$ & $5.0 \pm 1.3$ & $4.9 \pm 1.2$ & $4.8 \pm 1.3$ & $4.6 \pm 1.5$ & $<0.001$ \\
\hline LDL-C (mM) & $3.0 \pm 0.9$ & $2.9 \pm 0.9$ & $2.9 \pm 0.9$ & $2.6 \pm 0.9$ & $<0.001$ \\
\hline \multirow[t]{2}{*}{$\mathrm{HDL}-\mathrm{C}(\mathrm{mM})$} & $1.0 \pm 0.3$ & $1.0 \pm 0.3$ & $1.1 \pm 0.3$ & $1.0 \pm 0.3$ & \\
\hline & & & & & 0.110 \\
\hline ALT (IU/L) & $29(20-43)$ & $22(17-33)$ & $22(16-32)$ & $18(13-25)$ & $<0.001$ \\
\hline AST (IU/L) & $20(16-27)$ & $19(15-23)$ & $19(16-24)$ & $19(14-26)$ & 0.671 \\
\hline $\operatorname{Scr}(\mu \mathrm{M})$ & $59.2 \pm 19.6$ & $60.3 \pm 15.9$ & $60.0 \pm 15.7$ & $66.8 \pm 26.6$ & $<0.001$ \\
\hline $\operatorname{SUA}(\mu \mathrm{M})$ & $\begin{array}{l}344(283- \\
415)\end{array}$ & $\begin{array}{l}320(268- \\
387)\end{array}$ & $\begin{array}{l}313(274- \\
368)\end{array}$ & $\begin{array}{l}327(269- \\
384)\end{array}$ & 0.328 \\
\hline
\end{tabular}

Continuous variables were presented as means \pm SD or median (interquartile range).

\subsection{Associations between NFS and metabolic risk factors}

Next, the associations between NFS and other metabolic risk factors were further investigated. Analysis of Pearson's correlation showed that NFS was positively associated with age, duration of diabetes, BMI, SBP and Scr,while negatively associated with DBP, TG, TC, LDL-C, ALT and FPG (Table 2). Multiple stepwise linear regression analysis further revealed that sex, age, BMI, LDL-C, ALT, AST and HbA1c were independent determiners of NFS in type 2 diabetic subjects (Table 2). 


\begin{tabular}{|lllll|}
\hline & \multicolumn{2}{l}{ Univariate } & \multicolumn{2}{l|}{ Multivatiate } \\
\cline { 2 - 5 } & $\boldsymbol{r}$ & $\boldsymbol{p}$ & $\boldsymbol{\beta}$ & $\boldsymbol{p}$ \\
\hline Male & -0.001 & 0.959 & 0.249 & $<0.001$ \\
\hline Age (years) & 0.596 & $<0.001$ & 0.064 & $<0.001$ \\
\hline Duration & 0.297 & $<0.001$ & - & - \\
\hline BMI $\left(\mathrm{kg} / \mathrm{m}^{2}\right)$ & 0.152 & $<0.001$ & 0.09 & $<0.001$ \\
\hline SBP $(\mathrm{mmHg})$ & 0.115 & $<0.001$ & - & - \\
\hline DBP $(\mathrm{mmHg})$ & -0.08 & $<0.001$ & - & - \\
\hline TG $(\mathrm{mM})$ & -0.057 & 0.014 & - & - \\
\hline TC $(\mathrm{mM})$ & -0.113 & $<0.001$ & - & - \\
\hline LDL-C $(\mathrm{mM})$ & -0.15 & $<0.001$ & -0.122 & $<0.001$ \\
\hline HDL-C $(\mathrm{mM})$ & 0.21 & 0.37 & - & - \\
\hline FPG $(\mathrm{mM})$ & -0.055 & 0.018 & - & - \\
\hline HbA1C $(\%)$ & -0.024 & 0.295 & 0.036 & $<0.001$ \\
\hline ALT $(\mathrm{IU} / \mathrm{L})$ & -0.153 & $<0.001$ & -0.019 & $<0.001$ \\
\hline AST $(\mathrm{IU} / \mathrm{L})$ & -0.01 & 0.652 & 0.027 & $<0.001$ \\
\hline Scr $(\mu \mathrm{M})$ & 0.131 & $<0.001$ & - & - \\
\hline SUA $(\mu \mathrm{M})$ & 0.012 & 0.609 & - & - \\
\hline
\end{tabular}

Table 2

Associations between NFS and other metabolic risk factors

\subsection{Association of NFS with subclinical myocardial remodeling}

Subclinical myocardial remodeling of the subjects was evaluated by echocardiography. Parameters including left atrial dimension (LAD), interventricular septum thickness (IST), left ventricular end-diastolic diameter (LVEDD), left ventricular end-systolic diameter (LVESD) and left ventricular posterior wall thickness (LVPWT) were compared among the four groups according to the quartiles of NFS. As shown in Table 3, LAD, IST, LVEDD, LVESD and LVPWT were all gradually increased with the increment of NFS (all P for trend < 0.001). 
Table 3

Comparison of parameters of myocardial remodeling among groups according to quartiles of NFS

\begin{tabular}{|llllll|}
\hline & Q1 & Q2 & Q3 & Q4 & P for trend \\
\hline LAD $(\mathrm{mm})$ & $34.3 \pm 3.6$ & $35.1 \pm 3.1$ & $35.3 \pm 3.7$ & $36.7 \pm 4.0$ & $<0.001$ \\
\hline IST $(\mathrm{mm})$ & $8.2 \pm 1.0$ & $8.3 \pm 0.9$ & $8.5 \pm 1.0$ & $8.7 \pm 1.0$ & $<0.001$ \\
\hline LVEDD $(\mathrm{mm})$ & $47.7 \pm 4.3$ & $48.3 \pm 4.4$ & $48.5 \pm 4.0$ & $49.2 \pm 4.5$ & $<0.001$ \\
\hline $\operatorname{LVESD}(\mathrm{mm})$ & $31.0 \pm 4.0$ & $31.4 \pm 3.6$ & $31.7 \pm 3.9$ & $32.5 \pm 4.9$ & $<0.001$ \\
\hline LVPWT $(\mathrm{mm})$ & $8.1 \pm 0.8$ & $8.3 \pm 1.6$ & $8.4 \pm 0.8$ & $8.6 \pm 0.9$ & $<0.001$ \\
\hline
\end{tabular}

Linear regression analysis was further performed to determine the independent association between NFS and echocardiographic parameters. As shown in Table 4, NFS was positively associated with LAD, IST, LVEDD, LVESD and LVPWT in univariate analyses (Model 1). After adjustment for age, sex, BMI, SBP and DBP (model 2), NFS remain significantly correlated with the above echocardiographic parameters. Further adjustment for FPG, HbA1c, TG, TC, HDL-C, LDL-C (model 3) also did not significantly change the associations between NFS and LAD, IST, LVEDD, LVESD and LVPWT. 
Table 4

The association of NFS with subclinical myocardial remodeling

\begin{tabular}{|lllllll|}
\hline \multicolumn{1}{|c}{ Model 1 } & & Model 2 & & Model 3 & \\
\hline & $\beta(95 \% \mathrm{Cl})$ & $P$ & $\beta(95 \% \mathrm{Cl})$ & $P$ & $\beta(95 \% \mathrm{Cl})$ & $P$ \\
\hline LAD & 0.83 & $<0.001$ & 0.23 & 0.03 & 0.19 & 0.08 \\
& $(0.67,0.98)$ & & $(0.02,0.44)$ & & $(-0.03,0.40)$ & \\
\hline IST & 0.17 & $<0.001$ & 0.10 & $<0.001$ & 0.07 & 0.03 \\
& $(0.13,0.21)$ & & $(0.04,0.16)$ & & $(0.03,0.15)$ & \\
\hline LVEDD & 0.55 & $<0.001$ & 0.45 & $<0.001$ & 0.40 & $<0.01$ \\
& $(0.36,0.74)$ & & $(0.19,0.71)$ & & $(0.09,0.63)$ & \\
\hline LVESD & 0.52 & $<0.001$ & 0.47 & $<0.001$ & 0.39 & $<0.01$ \\
& $(0.33,0.70)$ & & $(0.22,0.73)$ & & $(0.14,0.65)$ & \\
\hline LVPWT & 0.16 & $<0.001$ & 0.12 & $<0.001$ & 0.09 & 0.01 \\
& $(0.12,0.21)$ & & $(0.06,0.18)$ & & $(0.02,0.15)$ & \\
\hline Model 1 is unadjusted. & & & & & \\
\hline Model 2 is adjusted for age, sex, BMl, SBP and DBP. & & \\
\hline Model 3 is further adjusted for FPG, HbA1C, TG, TC, LDL-C, HDL-C. & \\
\hline
\end{tabular}

\section{Discussion}

NAFLD is closely associated with CVD, while liver fibrosis is the main predictor of CVD and mortality. In the present study, we investigated the association of NFS, a noninvasive marker of liver advanced fibrosis in NAFLD, with subclinical myocardial remodeling in type 2 diabetic patients. It was found that NFS was independently associated with parameters of subclinical myocardial remodeling in patients with diabetes.

NFS was first used as a marker of advanced fibrosis in patients with NAFLD[18]. However, it was then found to be associated with CVD risk factors in different populations, regardless of the presence of NAFLD or not $[8,19]$. Consistently, we also observed positive associations of NFS with age, BMI, LDL-C and $\mathrm{HbA} 1 \mathrm{c}$. Moreover, previous studies have showed that NFS was related to CVD and left ventricular diastolic dysfunction in type 2 diabetic patients[11, 17]. In line with the previous studies, we observed an association between NFS and subclinical cardiovascular remodeling in type 2 diabetic patients in the present study. Altogether, NFS is a promising predictor for myocardial remodeling and CVD in type 2 diabetic patients. 
The potential mechanism linking NFS to myocardial remodeling is not fully established. One reason is that NFS is closely associated with other CVD risk factors, which contribute to the remodeling of heart. However, we found that NFS remained significantly associated with parameters of subclinical myocardial remodeling after adjustment of these risk factors, excluding the possibility that they mediate the association between NFS and myocardial remodeling. Thus, we could assume that there may be other mechanisms involved in the association of NFS with myocardial remodeling.

Recently, emerging evidence indicates that liver talks to heart by secreting a series of proteins, which are called hepatokines[20]. Hepatokines such as FGF21 can act on cardiac myocytes[21-22]. Abnormal secretion of hepatokines may contribute to the remodeling of heart in NAFLD. Moreover, inflammatory factors secreted by liver may also induce cardiovascular remodeling. However, the underlying mechanisms responsible for the remodeling of heart in NAFLD remain to be determined in future studies.

There are several limitations that require consideration in the present study. First, our study was crosssectional, which did not allow to make a cause-effect inference. Second, diagnosis of NAFLD was not included in the present study, precluding further analysis by stratifying the presence of NAFLD. However, NFS was previously shown to be associated with CVD and mortality in different population, regardless of the presence of NAFLD or not[8, 15]. Third, liver biopsy was not taken in the evaluation of fibrosis. Nevertheless, NFS is now widely used as a marker of liver advanced fibrosis[11].

\section{Conclusions}

In conclusion, the present study showed an association between NFS and subclinical myocardial remodeling in type 2 diabetic patients and it may be helpful for predicting or early detection of myocardial remodeling in diabetic patients.

\section{Abbreviations}

NAFLD: Nonalcoholic fatty liver disease; NFS: NAFLD fibrosis score; CAD: Coronary artery disease; BMI: Body mass index; HbA1c: Haemoglobin A1c; TC: Total cholesterol; TG: Triglyceride; LDL-C: Low density lipoprotein cholesterol; HDL-C: High density lipoprotein cholesterol; ALT: Alanine aminotransferase; AST: Aspartate aminotransferase; ScrखSerum creatinine.

\section{Declarations}

\section{Acknowledgements}

The authors thank all the staff and participants of this study for their important contributions.

\section{Authors' contributions}


Fan NG and Peng YD designed the study, analyzed the data and drafted the manuscript. Ding XY, Li N, Zhen Q, Gu LP, Fang F, Yan S, Pan L, Shen TT and Wang YF contributed to recruitment of patients. Zhang AF takes responsibility for the integrity of the data and the accuracy of the data analysis. All authors read and approved the final manuscript.

\section{Funding}

This work was supported by grants from the National Natural Science Foundation of China (81400785 and 81870596).

\section{Availability of data and materials}

The datasets used and/or analyzed during the current study are not publicly available but are available from the corresponding author on reasonable request.

\section{Ethics approval and consent to participate}

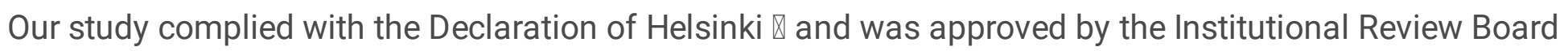
of Shanghai General Hospital affiliated to Shanghai Jiao Tong University School of Medicine. Informed written consents were obtained from all patients enrolled in this study.

\section{Consent for publication}

Not applicable.

\section{Competing interests}

We declare that we have no conflict of interest.

\section{Referance}

1. Hassan K, Bhalla V, El Regal ME, HH AK. Nonalcoholic fatty liver disease: a comprehensive review of a growing epidemic. World journal of gastroenterology. 2014; 20(34):12082-101.

2. Sheka AC, Adeyi O, Thompson J, Hameed B, Crawford PA, Ikramuddin S. Nonalcoholic Steatohepatitis: A Review. JAMA. 2020; 323(12):1175-83.

3. Kim JH, Moon JS, Byun SJ, Lee JH, Kang DR, Sung KC, et al. Fatty liver index and development of cardiovascular disease in Koreans without pre-existing myocardial infarction and ischemic stroke: a large population-based study. Cardiovascular diabetology. 2020; 19(1):51. 
4. Ismaiel A, Dumitraşcu DL. Cardiovascular Risk in Fatty Liver Disease: The Liver-Heart Axis-Literature Review. Frontiers in medicine. 2019; 6:202.

5. Simon TG, Bamira DG, Chung RT, Weiner RB, Corey KE. Nonalcoholic Steatohepatitis is Associated with Cardiac Remodeling and Dysfunction. Obesity (Silver Spring, Md). 2017; 25(8):1313-6.

6. Baratta F, Pastori D, Angelico F, Balla A, Paganini AM, Cocomello N, et al. Nonalcoholic Fatty Liver Disease and Fibrosis Associated With Increased Risk of Cardiovascular Events in a Prospective Study. Clinical gastroenterology and hepatology : the official clinical practice journal of the American Gastroenterological Association. 2019; S1542.

7. Tada T, Kumada T, Toyoda H, Mizuno K, Sone Y, Akita T, et al. Progression of liver fibrosis is associated with non-liver-related mortality in patients with nonalcoholic fatty liver disease. Hepatology communications. 2017; 1(9):899-910.

8. Chen Q, Li Q, Li D, Chen X, Liu Z, Hu G, et al. Association between liver fibrosis scores and the risk of mortality among patients with coronary artery disease. Atherosclerosis. 2020; 299:45-52.

9. Younossi ZM, Golabi P, de Avila L, Paik JM, Srishord M, Fukui N, Qiu Y, et al. The global epidemiology of NAFLD and NASH in patients with type 2 diabetes: A systematic review and meta-analysis. Journal of hepatology. 2019; 71(4):793-801.

10. Ciardullo S, Muraca E, Perra S, Bianconi E, Zerbini F, Oltolini A, et al. Screening for non-alcoholic fatty liver disease in type 2 diabetes using non-invasive scores and association with diabetic complications. BMJ open diabetes research \& care. 2020; 8(1): e000904.

11. Siddiqui MS, Yamada G, Vuppalanchi R, Van Natta M, Loomba R, Guy C, et al: Diagnostic Accuracy of Noninvasive Fibrosis Models to Detect Change in Fibrosis Stage. Clinical gastroenterology and hepatology : the official clinical practice journal of the American Gastroenterological Association. 2019; 17(9):1877-85.

12. Chen $Y, X u M$, Wang $T$, Sun $J$, Sun $W, X u B$, et al. Advanced fibrosis associates with atherosclerosis in subjects with nonalcoholic fatty liver disease. Atherosclerosis. 2015; 241(1):145-50.

13. Önnerhag $K$, Hartman $H$, Nilsson PM, Lindgren S. Non-invasive fibrosis scoring systems can predict future metabolic complications and overall mortality in non-alcoholic fatty liver disease (NAFLD). Scandinavian journal of gastroenterology. 2019; 54(3):328-34.

14. Kim D, Kim WR, Kim HJ, Therneau TM. Association between noninvasive fibrosis markers and mortality among adults with nonalcoholic fatty liver disease in the United States. Hepatology (Baltimore, Md). 2013; 57(4):1357-65.

15. Yoshihisa A, Sato Y, Yokokawa T, Sato T, Suzuki S, Oikawa M, et al. Liver fibrosis score predicts mortality in heart failure patients with preserved ejection fraction. ESC heart failure. 2018; 5(2):26270.

16. Unalp-Arida A, Ruhl CE. Liver fibrosis scores predict liver disease mortality in the United States population. Hepatology (Baltimore, Md). 2017; 66(1):84-95.

17. Lee H, Kim G, Choi YJ, Huh BW, Lee BW, Kang ES, et al. Association between Non-Alcoholic Steatohepatitis and Left Ventricular Diastolic Dysfunction in Type 2 Diabetes Mellitus. Diabetes \& 
metabolism journal. 2020; 44(2):267-76.

18. Sun W, Cui H, Li N, Wei Y, Lai S, Yang Y, et al. Comparison of FIB-4 index, NAFLD fibrosis score and BARD score for prediction of advanced fibrosis in adult patients with non-alcoholic fatty liver disease: A meta-analysis study. Hepatology research. 2016; 46(9):862-70.

19. Long MT, Pedley A, Massaro JM, Hoffmann U, Fox CS. The Association between Non-Invasive Hepatic Fibrosis Markers and Cardiometabolic Risk Factors in the Framingham Heart Study. PloS one. 2016; 11(6):e0157517.

20. Abdul-Wahed A, Gautier-Stein A, Casteras S, Soty M, Roussel D, Romestaing C, et al. A link between hepatic glucose production and peripheral energy metabolism via hepatokines. Molecular metabolism. 2014; 3(5):531-43.

21. Li S, Zhu Z, Xue M, Yi X, Liang J, Niu C, et al. Fibroblast growth factor 21 protects the heart from angiotensin II-induced cardiac hypertrophy and dysfunction via SIRT1. Biochimica et biophysica acta Molecular basis of disease. 2019; 1865(6):1241-52.

22. Shen $Y$, Ma X, Zhou J, Pan X, Hao Y, Zhou M, et al. Additive relationship between serum fibroblast growth factor 21 level and coronary artery disease. Cardiovascular diabetology. 2013; 12:124. 